\title{
COMPLEX HESSIAN EQUATION ON KAHLER MANIFOLD
}

\author{
ZUOLIANG HOU
}

\section{INTRODUCTION}

Let $(M, \omega)$ be a compact Kähler manifold of complex dimension $n$. In 1978, Yau Yau78 proved the famous Calabi-Yau conjecture by solving following complex Monge-Ampère equation on $M$

$$
(\omega+\sqrt{-1} \partial \bar{\partial} \varphi)^{n}=f \omega^{n}
$$

with positive function $f$. Later on, using tools from pluri-potential theory, Kołodziej [Ko198] studied the same equation with weaker smoothness assumption on $f$.

In this paper, we consider following complex Hessian equation on $(M, \omega)$ :

$$
\omega_{\varphi}^{k} \wedge \omega^{n-k}=(\omega+\sqrt{-1} \partial \bar{\partial} \varphi)^{k} \wedge \omega^{n-k}=f \omega^{n},
$$

where $k$ is a fixed integer between 2 and $n-1$, and $f$ is a non-negative function on $M$ satisfying the compatibility condition:

$$
\int_{M} f \omega^{n}=\int_{M} \omega^{n}
$$

Noticed that if $k=n$, equation (1) is just the complex Monge-Ampère equation, while if $k=1$, equation (1) becomes the Laplacian equation. So equation (1) is a generalization of both complex Monge-Ampère equation and Laplacian equation over compact Kähler manifold.

Similar nonlinear equations have been studied extensively by many authors, see [BT76, CNS84, CKNS85, CNS85, Li90, Tru95, GL96, TW99, Li04, Bło05] and the reference there.

The Main result of this paper is following

Theorem 1.1. Let $(M, \omega)$ be a compact Kähler manifold with nonnegative holomorphic bisectional curvature, and $f$ is a strictly positive smooth function, then equation (1) has smooth solution unique up to a constant.

Date: October 29, 2018. 
Our approach to Theorem 1.1 is similar to Yau's approach to the complex Monge-Ampère equation, i.e. continuity method and a priori estimate. By the standard theory of Krylov and Evans, it suffices to prove a priori $C^{2}$ estimate for equation (1). More precisely, we have

Proposition 1.2. If $\varphi$ solves equation (1) and $\sup _{M} \varphi=0$, then $\forall q>$ $2 n$,

$$
\|\varphi\|_{C^{0}} \leq C_{q}
$$

where $C_{q}$ is a constant depends on $(M, \omega), q$ and $\|f-1\|_{L^{q}}$.

Proposition 1.3. If $(M, \omega)$ has non-negative holomorphic bisectional curvature and $\varphi$ solves equation (1), then

$$
\|\nabla \varphi\|_{C^{0}(\omega)} \leq C_{1}
$$

where $C_{1}$ is a constant depends on $(M, \omega),\left\|f^{1 / k}\right\|_{C^{1}(\omega)}$ and $\operatorname{osc} \varphi$.

Proposition 1.4. If $(M, \omega)$ has non-negative holomorphic bisectional curvature and $\varphi$ solves equation (1), then

$$
\|\partial \bar{\partial} \varphi\|_{C^{0}(\omega)} \leq C_{2},
$$

where $C_{2}$ is a constant depends on $(M, \omega), \sup f, \inf \Delta_{\omega}\left(f^{1 / k}\right)$ and $\operatorname{osc} \varphi$.

Both Proposition 1.3 and Proposition 1.4 require non-negative holomorphic bisectional curvature for the underline Kähler manifold. However Yau's result requires no curvature condition, even though complex Monge-Ampère equation is worse than complex Hessian equation in certain sense, because Monge-Ampère equation is more nonlinear. One possible explanation is that the convex cone of positive real $(1,1)$ form is independent of the Kähler metric, while the convex cone of $k$ positive real $(1,1)$ form does depend on the Kähler metric. Besides, $\omega_{\varphi}$ being positive is a much stronger condition than $\omega_{\varphi}$ being $k$-positive. In fact, Yau used the positivity of $\omega_{\varphi}$ to control some third order terms in his proof of a priori $C^{2}$ estimate. Also noticed that Li [Li90] studied some nonlinear equations with certain structure conditions over compact Riemannian manifold which include the real Hessian equation as a special case. In Li's treatment, the non-negativity of sectional curvature is needed. Right now, We don't know whether the non-negativity of holomorphic bisectional curvature is an essential condition for the solvability of equation (1) or it is just a technical requirement.1]

\footnotetext{
${ }^{1}$ In a recent paper HMW08, we removed the curvature assumption imposed in this paper.
} 
We don't require the function $f$ to be strictly positive in both Proposition 1.3 and Proposition 1.4, i.e. equation (1) can be degenerate. However, in order to use the theory of Krylov and Evans to get higher regularity, we need the equation to be uniformly elliptic. Hence in Theorem 1.1, we require $f$ to be strictly positive.

Błocki [Bło05] studied the weak solution of complex Hessian equation over bounded domain in $\mathbb{C}^{n}$. The concept of weak solution and complex Hessian measure can be extended to the study of complex Hessian equation over compact Kähler manifold, and one can also study the corresponding potential theory.

We organize the rest of the paper as follows: in Section 2, we provide some necessary results on convex cones related to elementary symmetric functions; in Section 3. we study the uniqueness of solution and the $C^{0}$ estimate; in Section 4 and Section 5 , we derive the a priori $C^{1}$ and $C^{2}$ estimate respectively.

\section{Preliminary}

Let $S_{k}$ be the normalized $k$-th elementary symmetric function defined on $\mathbb{R}^{n}$ and let

$$
\Gamma_{k}=\left\{\lambda \in \mathbb{R}^{n} \mid S_{j}(\lambda)>0, j=1, \cdots, k\right\} .
$$

It is well known that $\Gamma_{k}$ is an open convex cone in $\mathbb{R}^{n}$. We call $\Gamma_{k}$ the $k$-positive cone in $\mathbb{R}^{n}$.

Proposition 2.1. For the $k$-positive cone $\Gamma_{k}$ in $\mathbb{R}^{n}$,

- $\Gamma_{n}=\left\{\lambda=\left(\lambda_{1}, \cdots, \lambda_{n}\right) \in \mathbb{R}^{n} \mid \lambda_{j}>0, j=1, \cdots, n\right\}$. We call $\Gamma_{n}$ the positive cone in $\mathbb{R}^{n}$.

- $\Gamma_{k}$ is the connected component of $\left\{\lambda \in \mathbb{R}^{n} \mid S_{k}(\lambda)>0\right\}$ containing $\Gamma_{n}$.

- $\forall \lambda \in \Gamma_{k}, \forall j=1, \cdots, n$,

$$
\frac{\partial S_{k}}{\partial \lambda_{j}}(\lambda)>0
$$

- (Newton inequalities) $\forall \lambda \in \mathbb{R}^{n}, \forall j=1, \cdots, n-1$,

$$
S_{j-1}(\lambda) S_{j+1}(\lambda) \leq S_{j}(\lambda)^{2} .
$$

- (Maclaurin inequalities) For $\lambda \in \Gamma_{k}$,

$$
0<S_{k}^{1 / k}(\lambda) \leq \cdots \leq S_{2}^{1 / 2}(\lambda) \leq S_{1}(\lambda) .
$$

- (Generalized Newton-Maclaurin inequalities) For $\lambda \in \Gamma_{k}$

$$
\left(\frac{S_{k}(\lambda)}{S_{l}(\lambda)}\right)^{\frac{1}{k-l}} \leq\left(\frac{S_{r}(\lambda)}{S_{s}(\lambda)}\right)^{\frac{1}{r-s}}
$$


where $0 \leq s<r, 0 \leq l<k, r \leq k$ and $s \leq l$.

- (Gårding inequality) Let $P_{k}$ be the complete polarization of $S_{k}$, then for $\Lambda^{(1)}, \cdots, \Lambda^{(k)} \in \Gamma_{k}$,

$$
P_{k}\left(\Lambda^{(1)}, \cdots, \Lambda^{(k)}\right) \geq S_{k}\left(\Lambda^{(1)}\right)^{1 / k} \cdots S_{k}\left(\Lambda^{(k)}\right)^{1 / k} .
$$

- $S_{k}^{1 / k}$ is concave on $\Gamma_{k}$.

- If $0 \leq l<k$, then $\left(S_{k} / S_{l}\right)^{1 /(k-l)}$ is concave on $\Gamma_{k}$.

The properties listed above are well known, for proof see CNS85, Går59. In this paper, we also need following result.

Lemma 2.2. Suppose $\lambda=\left(\lambda_{1}, \cdots, \lambda_{n}\right) \in \Gamma_{k}$ with

$$
\lambda_{1} \geq \lambda_{2} \geq \cdots \geq \lambda_{n},
$$

then

Moreover

$$
\frac{\partial S_{k}}{\partial \lambda_{1}} \leq \frac{\partial S_{k}}{\partial \lambda_{2}} \leq \cdots \leq \frac{\partial S_{k}}{\partial \lambda_{n}}
$$

$$
\lambda_{1} \frac{\partial S_{k}}{\partial \lambda_{1}} \geq \frac{k}{n} S_{k}
$$

Proof. Equation (9) is obvious, we will only prove equation (10). Let $\sigma_{k}$ be the $k$-th elementary symmetric function, and $\Lambda=\left\{\lambda_{1}, \cdots, \lambda_{n}\right\}$, then

$$
n \lambda_{1} \frac{\partial \sigma_{k}}{\partial \lambda_{1}}-k \sigma_{k}=(n-k) \lambda_{1} \sigma_{k-1}\left(\Lambda \backslash \lambda_{1}\right)-k \sigma_{k}\left(\Lambda \backslash \lambda_{1}\right)
$$

By Newton's Inequality for $\Lambda \backslash \lambda_{1}$,

$$
\lambda_{1} \geq S_{1}\left(\Lambda \backslash \lambda_{1}\right) \geq \frac{S_{k}\left(\Lambda \backslash \lambda_{1}\right)}{S_{k-1}\left(\Lambda \backslash \lambda_{1}\right)}=\frac{k \sigma_{k}\left(\Lambda \backslash \lambda_{1}\right)}{(n-k) \sigma_{k-1}\left(\Lambda \backslash \lambda_{1}\right)} .
$$

So

$$
n \lambda_{1} \frac{\partial S_{k}}{\partial \lambda_{1}} \geq k S_{k}
$$

Let $\mathcal{H}(n)$ be the set of $n \times n$ Hermitian matrices. We extend the definition of $S_{k}$ on $\mathbb{R}^{n}$ to $\mathcal{H}(n)$ by

$$
S_{k}(A)=S_{k}(\lambda(A))
$$

where $\lambda(A)$ is the eigenvalues of Hermitian matrix $A$, then $S_{k}$ is a homogeneous polynomial of degree $k$ on $\mathcal{H}(n)$ and

$$
\operatorname{det}\left(A+t I_{n}\right)=\sum_{j=0}^{n}\left(\begin{array}{l}
n \\
j
\end{array}\right) S_{j}(A) t^{n-j}
$$


We define the $k$-positive cone in $\mathcal{H}(n)$ by

$$
\Gamma_{k}(\mathcal{H}(n))=\left\{A \in \mathcal{H}(n) \mid S_{j}(A)>0, j=1, \cdots, k\right\} .
$$

Because of equation (11), it is easy to see that $S_{k}$ is invariant under the adjoint action of $U(n)$, hence $\Gamma_{k}(\mathcal{H}(n))$ is also $U(n)$-invariant. Besides, all the the properties listed in Proposition 2.1 are also true for $S_{k}$ defined on $\mathcal{H}(n)$. Especially, for any $A=\left(A_{\alpha \bar{\beta}}\right) \in \Gamma_{k}(\mathcal{H}(n))$, the matrix with entries given by

$$
F^{\alpha \bar{\beta}}=\frac{\partial \log S_{k}(A)}{\partial A_{\alpha \bar{\beta}}}
$$

is a positive definite Hermitian matrix.

Now consider a complex vector space $V$ of complex dimension $n$ with a fixed Hermitian metric $g$. Let $\omega$ be the Hermitian form of $g$. After fixing an unitary basis $\left\{\theta^{1}, \cdots, \theta^{n}\right\}$ for $V^{*}$, any real $(1,1)$ form $\chi$ can be written as

$$
\chi=\sqrt{-1} \chi_{\alpha \bar{\beta}} \theta^{\alpha} \wedge \overline{\theta^{\beta}}
$$

where $A_{\chi}=\left(\chi_{\alpha \bar{\beta}}\right)$ is a Hermitian matrix. We define the $k$-th Hermitian $S_{k}(\chi)$ of $\chi$ with respect to $\omega$ as

$$
S_{k}(\chi)=S_{k}\left(A_{\chi}\right)=S_{k}\left(\left(\chi_{\alpha \bar{\beta}}\right)\right) .
$$

The definition of $S_{k}$ is independent of the choice of unitary basis, in fact $S_{k}(\chi)$ can be defined without the use of unitary basis by

$$
\chi^{k} \wedge \omega^{n-k}=S_{k}(\chi) \omega^{n} .
$$

Let $\Lambda_{\mathbb{R}}^{1,1} V^{*}$ be the space of real $(1,1)$ form. We define the $k$-positive cone in $\Lambda_{\mathbb{R}}^{1,1} V^{*}$ by

$$
\Gamma_{k}\left(V^{1,1}\right)=\left\{\chi \in \Lambda_{\mathbb{R}}^{1,1} V^{*} \mid S_{j}(\chi)>0, j=1, \cdots, k\right\} .
$$

All the properties listed in Proposition 2.1 continue to be true for the $k$-th Hessian of real $(1,1)$ forms. Especially, for any $\chi_{1}, \cdots, \chi_{k} \in$ $\Gamma_{k}\left(V^{1,1}\right)$,

$$
\chi_{1} \wedge \cdots \wedge \chi_{k} \wedge \omega^{n-k}>0 .
$$

Let $(M, \omega)$ be a Kähler manifold with Kähler form $\omega$. The tangent space of $M$ at every point is a complex vector space with Hermitian metric, so the construction of $\Gamma_{k}\left(V^{1,1}\right)$ can be carried out pointwise on $M$, hence we get a distribution of open convex cones in the space of real $(1,1)$ form on $M$. Since the parallel transportation keeps the Kähler metric, so this distribution of convex cones is also invariant under the parallel transportation. For simplicity, we still use $\Gamma_{k}$ to denote these convex cones. 
Definition 2.3. A real $(1,1)$ form $\chi \in \Omega^{1,1}(M, \mathbb{R})$ is $k$-positive with respect to $\omega$, if $\chi \in \Gamma_{k}$.

Let $\mathcal{C}^{\infty}(M, \mathbb{R})$ be the set of real valued smooth functions on $M$. Denote

$$
\mathcal{P}_{k}(M, \omega)=\left\{\varphi \in \mathcal{C}^{\infty}(M, \mathbb{R}) \mid \omega_{\varphi}=\omega+\sqrt{-1} \partial \bar{\partial} \varphi \text { is } k \text {-positive }\right\} .
$$

Proposition 2.4. If $\varphi \in \mathcal{C}^{\infty}(M, \mathbb{R})$ solves (1), then $\varphi \in \mathcal{P}_{k}(M, \omega)$.

Proof. Since $\omega_{\varphi}$ is a positive form at the point where $\varphi$ achieves minimum, so $\omega_{\varphi} \in \Gamma_{n} \subset \Gamma_{k}$ at the minimum point. This together with the facts that $\Gamma_{k} \subset \mathcal{H}(n)$ is the connected component of $\left\{S_{k}>0\right\}$ containing $\Gamma_{n}$, and the distribution of these cones is invariant under parallel transportation shows that $\omega_{\varphi} \in \Gamma_{k}$ at every point, hence $\varphi \in \mathcal{P}_{k}(M, \omega)$.

Proposition 2.5. If $\varphi \in \mathcal{P}_{k}(M, \omega)$, then the operator

$$
\varphi \mapsto S_{k}\left(\omega_{\varphi}\right)=\frac{\omega_{\varphi}^{k} \wedge \omega^{n-k}}{\omega^{n}}
$$

is elliptic at $\varphi$.

\section{UNIQUENESS}

Suppose both $\varphi$ and $\psi$ solve equation (1), then

$$
\begin{aligned}
0 & =\int_{M}(\psi-\varphi)\left(\omega_{\psi}^{k} \wedge \omega^{n-k}-\omega_{\varphi}^{k} \wedge \omega^{n-k}\right) \\
& =\sum_{l=0}^{k-1} \int_{M}(\psi-\varphi) \sqrt{-1} \partial \bar{\partial}(\psi-\varphi) \wedge \omega_{\psi}^{l} \wedge \omega_{\varphi}^{k-1-l} \wedge \omega^{n-k} \\
& =-\sum_{l=0}^{k-1} \int_{M} \sqrt{-1} \partial(\psi-\varphi) \wedge \bar{\partial}(\psi-\varphi) \wedge \omega_{\psi}^{l} \wedge \omega_{\varphi}^{k-1-l} \wedge \omega^{n-k} \\
& \leq 0
\end{aligned}
$$

The last inequality is true because

$$
\sqrt{-1} \partial(\psi-\varphi) \wedge \bar{\partial}(\psi-\varphi) \in \Gamma_{n}, \quad \omega_{\varphi} \in \Gamma_{k} \quad \text { and } \quad \omega_{\psi} \in \Gamma_{k},
$$

hence by equation (13), for $l=0, \cdots, k-1$,

$$
\sqrt{-1} \partial(\psi-\varphi) \wedge \bar{\partial}(\psi-\varphi) \wedge \omega_{\psi}^{l} \wedge \omega_{\varphi}^{k-1-l} \wedge \omega^{n-k} \geq 0 .
$$

So $\partial(\psi-\varphi)=0$, and

$$
\omega_{\varphi}=\omega_{\psi} .
$$

Same positivity argument can be used to prove Proposition 1.2 by Yau's Moser iteration, see [Siu87] or [Tia00] for details. 


\section{4. $C^{1}$ estimate}

We will follow Błocki's approach in Bło07] to get the a priori $C^{1}$ estimate. But unlike Błocki, we will use the covariant derivative with respect to $\omega$ throughout this paper.

First let's fix some notation. Let $\left\{e_{1}, \cdots, e_{n}\right\}$ be a fixed unitary frame for $(M, \omega)$, and $\left\{\theta^{1}, \cdots, \theta^{n}\right\}$ be the duel frame. For $\varphi \in \mathcal{P}_{k}(M, \omega)$ with

$$
\sqrt{-1} \partial \bar{\partial} \varphi=\sqrt{-1} \varphi_{\alpha \bar{\beta}} \theta^{\alpha} \wedge \overline{\theta^{\beta}}
$$

let

$$
G^{\alpha \bar{\beta}}=F^{\alpha \bar{\beta}}\left(I_{n}+\left(\varphi_{\gamma \bar{\delta}}\right)\right),
$$

where $\left(F^{\alpha \bar{\beta}}\right)$ is the matrix-valued function defined on $\Gamma_{k}(\mathcal{H}(n))$ by equation (12). Let $\left(G_{\alpha \bar{\beta}}\right)$ be the inverse matrix of $\left(G^{\alpha \bar{\beta}}\right)$, then

$$
G=\sqrt{-1} G_{\alpha \bar{\beta}} \theta^{\alpha} \wedge \overline{\theta^{\beta}}
$$

induces a Hermitian metric $G$ on $M$, and this metric is independent of the choice of unitary frame.

Suppose $\varphi$ solves equation (11) with $\inf \varphi=0$ and $\sup \varphi=\operatorname{osc} \varphi=C_{0}$. Let

$$
B=\|\nabla \varphi\|_{\omega}^{2}=\varphi_{, \gamma} \varphi^{, \gamma} \quad \text { and } \quad A=\log B-h(\varphi),
$$

with $h(t)=\frac{1}{2} \log (2 t+1)$. Let $\Delta^{\prime}=G^{\alpha \bar{\beta}} \nabla_{\overline{e_{\beta}}} \nabla_{e_{\alpha}}$, then

$$
\Delta^{\prime} A=\frac{\Delta^{\prime} B}{B}-\frac{1}{B^{2}} G^{\alpha \bar{\beta}} B_{, \alpha} B_{, \bar{\beta}}-h^{\prime}(\varphi) \Delta^{\prime} \varphi-h^{\prime \prime}(\varphi) G^{\alpha \bar{\beta}} \varphi_{, \alpha} \varphi_{, \bar{\beta}}
$$

and

$$
\begin{aligned}
\Delta^{\prime} B & =G^{\alpha \bar{\beta}}\left(\varphi^{, \gamma}{ }_{\alpha \bar{\beta}} \varphi_{, \gamma}+\varphi^{, \gamma}{ }_{\alpha} \varphi_{, \gamma \bar{\beta}}+\varphi^{, \gamma}{ }_{\bar{\beta}} \varphi_{, \gamma \alpha}+\varphi^{, \gamma} \varphi_{, \gamma \alpha \bar{\beta}}\right) \\
& \geq G^{\alpha \bar{\beta}}\left(\varphi^{, \gamma}{ }_{\alpha \bar{\beta}} \varphi_{, \gamma}+\varphi^{, \gamma} \varphi_{, \gamma \alpha \bar{\beta}}\right) .
\end{aligned}
$$

By Ricci identity

$$
\varphi^{, \gamma}{ }_{\alpha \bar{\beta}}=\varphi_{, \alpha \bar{\beta}}{ }^{\gamma} \quad \text { and } \quad \varphi_{, \gamma \alpha \bar{\beta}}=\varphi_{, \alpha \bar{\beta} \gamma}+\varphi_{, \eta} R_{\alpha \gamma \bar{\beta}}^{\eta}
$$

Since $\varphi$ solves equation (11), so

$$
G^{\alpha \bar{\beta}} \varphi_{, \alpha \bar{\beta} \gamma}=(\log f)_{, \gamma} \quad \text { and } \quad G^{\alpha \bar{\beta}} \varphi_{, \alpha \bar{\beta}^{\gamma}}=(\log f)^{, \gamma} .
$$

Hence

$$
\Delta^{\prime} B \geq 2 \operatorname{Re}\langle\nabla(\log f), \nabla \varphi\rangle_{\omega}+G^{\alpha \bar{\beta}} \varphi_{, \eta} \varphi^{, \gamma} R_{\alpha \gamma \bar{\beta}}^{\eta}
$$

If $(M, \omega)$ has non-negative holomorphic bisectional curvature, then

$$
G^{\alpha \bar{\beta}} \varphi_{, \eta} \varphi^{, \gamma} R_{\alpha \gamma \bar{\beta}}^{\eta} \geq 0
$$

therefore

$$
\Delta^{\prime} B \geq 2 \operatorname{Re}\langle\nabla(\log f), \nabla \varphi\rangle_{\omega}
$$


Noticed that $S_{k}$ is homogeneous of polynomial of degree $k$, so we have

$$
\Delta^{\prime} \varphi=G^{\alpha \bar{\beta}} \varphi_{, \alpha \bar{\beta}}=k-\operatorname{tr}_{\omega} G .
$$

If $A$ achieves maximum at point $p$, then at point $p, \nabla A=0$, i.e.

$$
\frac{B_{, \alpha}}{B}=h^{\prime}(\varphi) \varphi_{, \alpha} \quad \text { and } \quad \frac{B_{, \bar{\beta}}}{B}=h^{\prime}(\varphi) \varphi_{, \bar{\beta}} .
$$

Hence at the maximum point $p$,

$$
\Delta^{\prime} A \geq \frac{2}{B} \operatorname{Re}\langle\nabla(\log f), \nabla \varphi\rangle-\left(h^{\prime \prime}+h^{\prime 2}\right) G^{\alpha \bar{\beta}} \varphi_{, \alpha} \varphi_{, \bar{\beta}}-k h^{\prime}+h^{\prime} \operatorname{tr}_{\omega} G .
$$

Since $\varphi$ takes value between 0 and $C_{0}$, so

$$
\frac{1}{2 C_{0}+1} \geq h^{\prime} \geq 1 \quad \text { and } \quad-h^{\prime \prime}-h^{2} \geq \frac{1}{\left(2 C_{0}+1\right)^{2}} .
$$

Therefore

$$
\Delta^{\prime} A \geq \frac{2}{B} \operatorname{Re}\langle\nabla(\log f), \nabla \varphi\rangle+\frac{G^{\alpha \bar{\beta}} \varphi_{, \alpha} \varphi_{, \bar{\beta}}}{\left(2 C_{0}+1\right)^{2}}-k+\frac{\operatorname{tr}_{\omega} G}{2 C_{0}+1} .
$$

If the eigenvalue of $\omega+\sqrt{-1} \partial \bar{\partial} \varphi$ with respect to $\omega$ is $\left(\lambda_{1}, \cdots \lambda_{n}\right)$, then

$$
\operatorname{tr}_{\omega} G=k \frac{S_{k-1}(\lambda)}{S_{k}(\lambda)} \text { and } f=S_{k}(\lambda) .
$$

Noticed

$$
|\nabla \log f|=\frac{k\left|\nabla\left(f^{1 / k}\right)\right|}{f^{1 / k}} \leq\left|\nabla\left(f^{1 / k}\right)\right| \operatorname{tr}_{\omega} G,
$$

here we've used the Maclaurin Inequality

$$
\frac{1}{f^{1 / k}}=\frac{1}{S_{k}^{1 / k}(\lambda)} \leq \frac{S_{k-1}(\lambda)}{S_{k}(\lambda)}=\frac{1}{k} \operatorname{tr}_{\omega} G
$$

So at the maximum point $p$,

$$
0 \geq\left(\frac{1}{2 C_{0}+1}-\frac{2\left|\nabla\left(f^{1 / k}\right)\right|}{|\nabla \varphi|}\right) \operatorname{tr}_{\omega} G+\frac{1}{\left(2 C_{0}+1\right)^{2}} G^{\alpha \bar{\beta}} \varphi_{, \alpha} \varphi_{, \bar{\beta}}-k
$$

We may also assume that

$$
\|\nabla \varphi\| \leq \frac{1}{2\left(2 C_{0}+1\right)}\left\|\nabla\left(f^{1 / k}\right)\right\|
$$

therefore at $p$,

$$
k \geq \frac{1}{\left(2 C_{0}+1\right)^{2}} G^{\alpha \bar{\beta}} \varphi_{, \alpha} \varphi_{, \bar{\beta}}+\frac{1}{2\left(2 C_{0}+1\right)} \operatorname{tr}_{\omega} G .
$$

So at the maximum point $p$,

$$
\operatorname{tr}_{\omega} G \leq C, \quad \text { and } \quad G^{\alpha \bar{\beta}} \varphi_{, \alpha} \varphi_{, \bar{\beta}} \leq C
$$


where $C$ is a constant depends on $C_{0}$ only. By the Generalized NewtonMaclaurin Inequality,

$$
S_{1}(\lambda) \leq S_{k}(\lambda)\left(\frac{S_{k-1}(\lambda)}{S_{k}(\lambda)}\right)^{k-1} \leq f\left(\frac{\operatorname{tr}_{\omega} G}{k}\right)^{k-1}
$$

$S_{1}(\lambda)$ is bounded by constant depends on $C_{0}$ and $\sup f$ at point $p$. Noticed that $\forall \lambda \in \mathbb{R}^{n}$,

$$
\sum_{j} \lambda_{j}^{2}=\left(\sum_{j} \lambda_{j}\right)^{2}-\sum_{i \neq j} \lambda_{i} \lambda_{j}=\left(n S_{1}(\lambda)\right)^{2}-n(n-1) S_{2}(\lambda) .
$$

Since $\lambda \in \Gamma_{k}$ with $k \geq 2$, so $S_{2}(\lambda) \geq 0$ and

$$
\sup _{j}\left|\lambda_{j}\right| \leq n S_{1}(\lambda) \text {. }
$$

Therefor at the maximum point $p$, the eigenvalue of $\omega_{\varphi}$ with respect to $\omega$ is bounded. If we further assume that $\lambda_{1} \geq \cdots \geq \lambda_{n}$, then the smallest eigenvalue of matrix $\left(G^{\alpha \bar{\beta}}\right)$ is $\frac{1}{S_{k}(\lambda)} \frac{\partial S_{k}(\lambda)}{\partial \lambda_{1}}$, which can be bounded from below because of Lemma 2.2 ,

$$
\frac{1}{S_{k}} \frac{\partial S_{k}}{\partial \lambda_{1}} \geq \frac{k}{n \lambda_{1}}
$$

Combine equation (21) and (22), $\nabla \varphi$ is bounded at the maximum point of $A$, therefore $A$ is bounded everywhere, hence $\nabla \varphi$ is also bounded everywhere, and this finishes the proof of Proposition 1.3.

\section{5. $C^{2}$ estimate}

Same as in Section 4, we will use maximum principle to get some $a$ priori estimate in this section. We can keep using the Hermitian metric introduced by equation (14). But in order to get better regularity result, we will introduce a new Hermitian metric. For $A=\left(A_{\alpha \bar{\beta}}\right) \in$ $\Gamma_{k}(\mathcal{H}(n))$, denote

$$
\tilde{F}^{\alpha \bar{\beta}}(A)=\frac{\partial S_{k}^{1 / k}}{\partial A_{\alpha \bar{\beta}}},
$$

then $\left(\tilde{F}^{\alpha \bar{\beta}}(A)\right)$ is positive definite Hermitian matrix. Using the same frame $\left(e_{1}, \cdots, e_{n}\right)$ and co-frame $\left(\theta^{1}, \cdots, \theta^{n}\right)$ as in Section 4 , for $\varphi \in$ $\mathcal{P}_{k}(M, \omega)$ with

$$
\partial \bar{\partial} \varphi=\varphi_{, \alpha \bar{\beta}} \theta^{\alpha} \wedge \overline{\theta^{\beta}}
$$

let

$$
H^{\alpha \bar{\beta}}=\tilde{F}^{\alpha \bar{\beta}}\left(I_{n}+\left(\varphi_{, \alpha \bar{\beta}}\right)\right),
$$

and $\left(H_{\alpha \bar{\beta}}\right)=\left(H^{\alpha \bar{\beta}}\right)^{-1}$, then

$$
H=\sqrt{-1} H_{\alpha \bar{\beta}} \theta^{\alpha} \wedge \bar{\theta}
$$


induces a Hermitian metric on $M$.

Let $\tilde{\Delta}=H^{\alpha \bar{\beta}} \nabla_{\overline{e_{\beta}}} \nabla_{e_{\alpha}}$, then

$$
\tilde{\Delta}(n+\Delta \varphi)=H^{\alpha \bar{\beta}} \varphi_{, \gamma \bar{\gamma} \alpha \bar{\beta}} .
$$

From the equation

$$
S_{k}^{1 / k}\left(I_{n}+\left(\varphi_{\alpha \bar{\beta}}\right)\right)=f^{1 / k}
$$

one get

$$
H^{\alpha \bar{\beta}} \varphi_{, \alpha \bar{\beta} \gamma \bar{\gamma}} \geq \Delta\left(f^{1 / k}\right) .
$$

Here we've used the concavity of $S_{k}^{1 / k}$. By Ricci identity,

$$
\varphi_{, \alpha \bar{\beta} \gamma \bar{\gamma}}=\varphi_{, \gamma \bar{\gamma} \alpha \bar{\beta}}+\varphi_{, \xi \bar{\beta}} R_{\alpha \gamma \bar{\gamma}}^{\xi}-\varphi_{, \xi \bar{\gamma}} R_{\gamma \alpha \bar{\beta}}^{\xi}
$$

So

$$
\tilde{\Delta}(n+\Delta \varphi) \geq \Delta f+H^{\alpha \bar{\beta}} \varphi_{, \xi \bar{\gamma}} R_{\gamma \alpha \bar{\beta}}^{\xi}-H^{\alpha \bar{\beta}} \varphi_{, \xi \bar{\beta}} R_{\alpha \gamma \bar{\gamma}}^{\xi}
$$

Choose a unitary frame so that $\varphi_{\alpha \bar{\beta}}$ is diagonal matrix, then

$$
\begin{aligned}
& H^{\alpha \bar{\beta}} \varphi_{, \xi \bar{\gamma}} R_{\gamma \alpha \bar{\beta}}^{\xi}-H^{\alpha \bar{\beta}} \varphi_{, \xi \bar{\beta}} R_{\alpha \gamma \bar{\gamma}}^{\xi} \\
= & \sum_{\alpha, \gamma}\left(H^{\alpha \bar{\alpha}} \varphi_{, \gamma \bar{\gamma}} R \bar{\gamma} \gamma \alpha \bar{\alpha}-H^{\alpha \bar{\alpha}} \varphi_{, \alpha \bar{\alpha}} R_{\bar{\alpha} \alpha \gamma \bar{\gamma}}\right) \\
= & \sum_{\alpha, \gamma} H^{\alpha \bar{\alpha}} R_{\bar{\gamma} \gamma \alpha \bar{\alpha}}\left(\varphi_{, \gamma \bar{\gamma}}-\varphi_{, \alpha \bar{\alpha}}\right) \\
= & \sum_{\alpha<\gamma} R_{\bar{\gamma} \gamma \alpha \bar{\alpha}}\left(\varphi_{, \gamma \bar{\gamma}}-\varphi_{, \alpha \bar{\alpha}}\right)\left(H^{\alpha \bar{\alpha}}-H^{\gamma \bar{\gamma}}\right)
\end{aligned}
$$

If $\omega_{\varphi}$ is diagonalized as $\left(\lambda_{1}, \cdots, \lambda_{n}\right)$ with respect to $\omega$, then

$$
\varphi_{\alpha \bar{\alpha}}=\lambda_{\alpha}-1 \quad \text { and } \quad H^{\alpha \bar{\alpha}}=\frac{1}{k} S_{k}^{\frac{1}{k}-1} \frac{\partial S_{k}}{\partial \lambda_{\alpha}} .
$$

Hence

$$
\varphi_{, \alpha \bar{\alpha}} \geq \varphi_{, \gamma \bar{\gamma}} \quad \Rightarrow \quad \lambda_{\alpha} \geq \lambda_{\gamma} \quad \Rightarrow \quad H^{\alpha \bar{\alpha}} \leq H^{\gamma \bar{\gamma}}
$$

therefore

$$
\left(\varphi_{, \gamma \bar{\gamma}}-\varphi_{, \alpha \bar{\alpha}}\right)\left(H^{\alpha \bar{\alpha}}-H^{\gamma \bar{\gamma}}\right) \geq 0 .
$$

If $(M, \omega)$ has non-negative holomorphic bisectional curvature, i.e.

$$
R_{\bar{\gamma} \gamma \alpha \bar{\alpha}} \geq 0,
$$

then

$$
H^{\alpha \bar{\beta}} \varphi_{, \xi \bar{\gamma}} R_{\gamma \alpha \bar{\beta}}^{\xi}-H^{\alpha \bar{\beta}} \varphi_{, \xi \bar{\beta}} R_{\alpha \gamma \bar{\gamma}}^{\xi} \geq 0
$$

and

$$
\tilde{\Delta}(n+\Delta \varphi) \geq \Delta\left(f^{1 / k}\right) .
$$


Consider $n+\Delta \varphi-\varphi$. At the point where $n+\Delta \varphi+\varphi$ achieves the maximum,

$$
0 \geq \tilde{\Delta}(n+\Delta \varphi-\varphi) \geq \Delta\left(f^{1 / k}\right)-f^{1 / k}+\operatorname{tr}_{\omega} H .
$$

Hence at the maximum point

$$
\operatorname{tr}_{\omega} H \leq f^{1 / k}-\Delta\left(f^{1 / k}\right) \leq C
$$

where $C$ is a constant depends on sup $f$ and inf $\Delta\left(f^{1 / k}\right)$. Noticed that

$$
\operatorname{tr}_{\omega} H=k S_{k}^{\frac{1}{k}} \frac{S_{k-1}}{S_{k}}
$$

and

$$
n+\Delta \varphi=S_{1}(\lambda) \leq S_{k}\left(\frac{S_{k-1}}{S_{k}}\right)^{k-1}=S_{k}^{\frac{1}{k}}\left(S_{k}^{\frac{1}{k}} \frac{S_{k-1}}{S_{k}}\right)^{k-1},
$$

so by bounding $\operatorname{tr}_{\omega} H$, we also bound $n+\Delta \varphi$ by constant depends on $\sup f$ and $\Delta\left(f^{1 / k}\right)$ at the maximum point of $n+\Delta \varphi-\varphi$. Therefore get a global bound for $n+\Delta \varphi$. Then by equation (23), we also get global bound for the eigenvalues of $\omega_{\varphi}$ with respect to $\omega$, i.e.

$$
\|\partial \bar{\partial} \varphi\|_{C^{0}(\omega)} \leq C_{2}
$$

with $C_{2}$ depends on $\sup f, \inf \Delta\left(f^{1 / k}\right)$ and $\operatorname{osc} \varphi$.

\section{REFERENCES}

[Bło05] Zbigniew Błocki. Weak solutions to the complex Hessian equation. Ann. Inst. Fourier (Grenoble), 55(5):1735-1756, 2005.

[Bło07] Zbigniew Błocki. A gradient estimate in the calabi-yau theorem. preprint, 2007.

[BT76] Eric Bedford and B. A. Taylor. The Dirichlet problem for a complex Monge-Ampère equation. Invent. Math., 37(1):1-44, 1976.

[CKNS85] L. Caffarelli, J. J. Kohn, L. Nirenberg, and J. Spruck. The Dirichlet problem for nonlinear second-order elliptic equations. II. Complex Monge-Ampère, and uniformly elliptic, equations. Comm. Pure Appl. Math., 38(2):209-252, 1985.

[CNS84] L. Caffarelli, L. Nirenberg, and J. Spruck. The Dirichlet problem for nonlinear second-order elliptic equations. I. Monge-Ampère equation. Comm. Pure Appl. Math., 37(3):369-402, 1984.

[CNS85] L. Caffarelli, L. Nirenberg, and J. Spruck. The Dirichlet problem for nonlinear second-order elliptic equations. III. Functions of the eigenvalues of the Hessian. Acta Math., 155(3-4):261-301, 1985.

[Går59] Lars Gårding. An inequality for hyperbolic polynomials. J. Math. Mech., 8:957-965, 1959.

[GL96] Bo Guan and Yan Yan Li. Monge-Ampère equations on Riemannian manifolds. J. Differential Equations, 132(1):126-139, 1996.

[HMW08] Zuoliang Hou, Xi-Nan Ma, and Damin Wu. Complex hessian equations on a compact kähler manifold. preprint, 2008. 
[Koł98] Sławomir Kołodziej. The complex Monge-Ampère equation. Acta Math., 180(1):69-117, 1998.

[Li90] Yan Yan Li. Some existence results for fully nonlinear elliptic equations of Monge-Ampère type. Comm. Pure Appl. Math., 43(2):233-271, 1990.

[Li04] Song-Ying Li. On the Dirichlet problems for symmetric function equations of the eigenvalues of the complex Hessian. Asian J. Math., 8(1):87106, 2004.

[Siu87] Yum Tong Siu. Lectures on Hermitian-Einstein metrics for stable bundles and Kähler-Einstein metrics, volume 8 of DMV Seminar. Birkhäuser Verlag, Basel, 1987.

[Tia00] Gang Tian. Canonical metrics in Kähler geometry. Lectures in Mathematics ETH Zürich. Birkhäuser Verlag, Basel, 2000. Notes taken by Meike Akveld.

[Tru95] Neil S. Trudinger. On the Dirichlet problem for Hessian equations. Acta Math., 175(2):151-164, 1995.

[TW99] Neil S. Trudinger and Xu-Jia Wang. Hessian measures. II. Ann. of Math. (2), 150(2):579-604, 1999.

[Yau78] Shing Tung Yau. On the Ricci curvature of a compact Kähler manifold and the complex Monge-Ampère equation. I. Comm. Pure Appl. Math., 31(3):339-411, 1978.

Mathematics Department, Columbia University, New York, NY 10027

E-mail address: hou@math.columbia.edu 